\title{
The profiles of the aromatic infrared bands explained with molecular carriers
}

\author{
C. Pech ${ }^{1}$, C. Joblin ${ }^{1}$, and P. Boissel ${ }^{2}$ \\ 1 Centre d'Étude Spatiale des Rayonnements, CNRS-UPS, BP 4346, 31028 Toulouse Cedex 04, France \\ 2 Laboratoire de Chimie Physique, Université Paris Sud, Bât 350, 91405 Orsay Cedex, France
}

Received 29 September 2000 / Accepted 28 February 2002

\begin{abstract}
Using a compilation of the photophysical properties of molecules such as coronene, $\mathrm{C}_{24} \mathrm{H}_{12}$, we have constructed a numerical model to calculate the IR emission spectrum of a population of polycyclic aromatic hydrocarbon (PAH) species subjected to UV photons. The model enables us to calculate not only the energy emitted in the IR bands but also the detailed band profiles. The calculated spectrum for a PAH size distribution following $N_{\mathrm{C}}^{-3.5}$ with $N_{\mathrm{Cmin}}=30$ was found to provide a good match to the Aromatic Infrared Bands (AIBs) observed in the planetary nebula IRAS $21282+5050$. The match is especially good for the intensities and for the profiles of the 6.2 and $11.3 \mu \mathrm{m}$ bands. This work provides a coherent picture of the AIB problem supporting the PAH molecular hypothesis. It further underlines the poor spectral diversity contained in the AIB spectrum putting new constraints on the nature of the emitting population.
\end{abstract}

Key words. infrared: ISM - ISM: lines and bands - line: profiles - ISM: general

\section{Introduction}

Thanks to the European satellite Infrared Space Observatory (ISO $)^{1}$, the well-known "Unidentified" Infrared Bands (UIBs) at 3.3, 6.2, 7.7, 8.6, 11.3 and $12.7 \mu \mathrm{m}$ have been observed in many regions of the interstellar medium (ISM) in our galaxy, such as high-latitude cirrus, reflection nebulae, planetary nebulae and HII regions as well as in external galaxies. These bands are the signatures of $\mathrm{CC}$ and $\mathrm{CH}$ bonds of hydrogenated aromatic structures, which has lead to a more recent re-naming as Aromatic IR Bands (AIBs). Several candidates have been proposed: polycyclic aromatic hydrocarbon molecules (PAHs) (Léger \& Puget 1984; Allamandola et al. 1989) and various carbonaceous grains such as coals (Papoular et al. 1989), hydrogenated amorphous carbons (Borghesi et al. 1987) and quenched carbonaceous composites (Sakata et al. 1987). The AIBs were observed in different environments with UV flux ranging from 1 to $10^{5}$ times the average value in the solar neighbourhood (Boulanger 1999). The overall spectral shape was

Send offprint requests to: C. Joblin, e-mail: joblin@cesr.fr

${ }^{1}$ ISO is an ESA project with instruments funded by ESA Member States (especially the PI countries: France, Germany, The Netherlands and the UK) and with the participation of ISAS and NASA. found to be constant and the band intensities were seen to scale with the intensity of the UV radiation field. This clearly rules out emission from grains at thermal equilibrium but is fully consistent with a transient heating following the absorption of a single UV photon. This excitation mechanism, first invoked by Andriesse (1978) and Sellgren (1984), requires the carriers to be very small. Recently, Cook et al. (1998) have shown, in the laboratory, that UVexcited PAHs emit in the 3-15 $\mu \mathrm{m}$ range and that the observed band widths are comparable to those of the AIBs. The IR emission spectrum of a PAH size distribution exposed to the radiation field of stars has been calculated by several authors. In particular, Schutte et al. (1993) have studied the influence on the emergent spectrum of the PAH photophysical properties such as the oscillator strengths of the IR active modes. They derived a generic spectrum for interstellar PAHs (standard model). This spectrum consists of bands at frequencies corresponding to the AIBs and oscillator strengths that have been adjusted to fit the observed spectra. These oscillator strengths differ from those of neutral PAHs but seem to be consistent with interstellar PAHs being ionized. Using a different approach, Cook \& Saykally (1998) calculated the spectrum from a collection of PAHs whose IR properties were measured in the laboratory. The simulated spectrum was obtained by using the spectral characteristics (frequencies and oscillator strengths) of each species in the mixture. 
A single emission temperature was considered to simplify the calculations. Mean band widths derived by Cook et al. (1998) from measurements on UV-excited perylene $\mathrm{C}_{20} \mathrm{H}_{12}$ and coronene $\mathrm{C}_{24} \mathrm{H}_{12}$ were used. The authors came to the same conclusion that $\mathrm{PAH}$ cations are better candidates than neutrals to account for the AIBs. However, they showed that the small sizes that were considered in the calculations (larger PAH in the mixture: $\mathrm{C}_{24} \mathrm{H}_{12}$ ) have larger band widths than the AIBs. The generic spectrum of Schutte et al. (1993) is therefore likely to be dominated by larger PAHs, although the spectral characteristics of such species are presently not known.

In this paper, we combine the approach presented by Schutte et al. (1993) and that of Cook \& Saykally (1998) to calculate the emission spectrum of a PAH population containing sizes of up to two hundred carbon atoms and subjected to the radiation field of a hot star. Photophysical properties measured on small PAHs were extrapolated to larger species (cf. Sect. 2.3). They include the IR band oscillator strengths, the band frequencies and widths and their evolution with temperature (cf. Sect. 2.3.3 (b)). Emission spectra were calculated by adding up, for a given initial internal energy (e.g. energy of the absorbed UV photon), the various contributions during the cooling of a given isolated PAH. Integration is then performed over the distribution of $\mathrm{PAH}$ sizes and the distribution of absorbed stellar photons. In this paper, we present the particular case of the planetary nebula IRAS $21282+5050$ for which a nice mid-IR spectrum was measured with the ISO Short Wavelength Spectrometer (observations by M. Jourdain de Muizon, L. d'Hendecourt, A. Heras and collaborators; cf. Joblin et al. 2000). This object was also selected because of the detection by Geballe et al. (1994) of the $1.68 \mu \mathrm{m}$ band, the overtone of the $3.3 \mu \mathrm{m}$ band. As discussed in Sect. 3.2, the intensity of this band can be used to constrain the minimum size of PAHs.

Our calculations based on the thermal model (Léger et al. 1989) are similar to those performed by Schutte et al. but they also consider the detailed profiles of the emitted bands which further constrain the model. Barker et al. (1987) first suggested that the asymmetry of the AIBs and in particular that of the 6.2 and $11.3 \mu \mathrm{m}$ bands is related to the anharmonicity of the modes. Anharmonic effects have been carefully described and included in the calculations. The calculated profiles are presented in Sect. 3 and compared to the observations.

The molecular nature of the AIB spectrum is then discussed. Implications for the nature of the carriers of the bands are then given (Sect. 3.3).

\section{Modelling the IR emission spectrum of "interstellar PAHs"}

The photophysics of interstellar PAHs have been described for example by Léger et al. (1989). After the absorption of an UV photon, the system follows a rapid evolution towards the ground electronic state (internal conversion). The energy is redistributed in the vibrational modes by the Intramolecular Vibrational Redistribution (IVR) mechanism, and the isolated excited species then cool down by emitting in their IR active modes. Some of the energy can also be lost through electronic fluorescence (Poincaré fluorescence; Léger et al. 1988). The contribution of the electronic fluorescence to the cooling process was found to be about $10 \%$ for the small cation coronene $\mathrm{C}_{24} \mathrm{H}_{12}^{+}$and to become negligible for larger $\mathrm{PAH}$ species. This mechanism was therfore not considered in the calculations.

\subsection{Infrared vibrational emission and cooling}

Assuming a statistical distribution of the energy among the vibrational modes, the infrared emission rate $k_{\mathrm{IR}, \mathrm{v}}^{i}$ of the mode $i$ in the transition $v \rightarrow v-1$ is given by:

$k_{\mathrm{IR}, v}^{i}=A_{i}^{v, v-1} \times \frac{\rho^{*}\left(U-v h \nu_{i}\right)}{\rho(U)}$

$A_{i}^{v, v-1}$ being the Einstein coefficient of spontaneous emission in the $v \rightarrow v-1$ transition, $\rho(U)$ the density of states and $\rho^{*}\left(U-v h \nu_{i}\right)$ the density of states for all modes except the $i$ emitting mode.

It has been shown (Léger et al. 1989; Schutte et al. 1993) that the thermal approximation can be used to describe the infrared cooling of PAHs. In this approximation, the emission in a given vibrational mode from a PAH of internal energy $U$ is calculated as the average emission of an oscillator connected to a thermal bath. This model assumes that, the energy content of the other modes being large enough, the PAH itself can be considered as a heat bath at the temperature $T$ which corresponds to an average energy $U$ of the molecule. Expressed in terms of specific heat, this can be written as:

$U(T)=\int_{0}^{T} C\left(T^{\prime}\right) \mathrm{d} T^{\prime}$.

In the case of an assembly of harmonic (or nearly harmonic) oscillators, the integrated form is readily obtained:

$U(T)=\sum_{i=1}^{s} \frac{h \nu_{i}}{\exp \left(h \nu_{i} / k T\right)-1}$

with $s$ being the total number of vibrational modes in the molecule.

The emission rate for the transition $v \rightarrow v-1$ of the mode $i$ is then given by:

$k_{\mathrm{IR}, v}^{i}=v \times A_{i}^{1,0} \times \exp \left(-v h \nu_{i} / k T\right) \times\left(1-\exp \left(-h \nu_{i} / k T\right)\right)$.

The summation of Eq. (4) over all the $v \rightarrow v-1$ transitions gives the expression of the total IR emission rate of the mode $i$ as:

$k_{\mathrm{IR}}^{i}=\sum_{v} k_{\mathrm{IR}, v}^{i}=A_{i}^{1,0} \times\left[\exp \left(h \nu_{i} / k T\right)-1\right]^{-1}$.

\subsection{Line profiles}

Several mechanisms can contribute to the broadening of the IR bands emitted by PAHs during their cooling. 


\subsubsection{Homogeneous band width}

The homogeneous IR band width of a vibrationnally excited PAH is governed by IVR. Driven by the coupling between states, IVR is effective at vibrational energies larger than $\sim 2000 \mathrm{~cm}^{-1}$ in the case of PAHs (Felker \& Zewail 1984). It leads to a lifetime of the levels much shorter than the radiative lifetime $\left(10^{-11} \mathrm{~s}\right.$ compared to $10^{-7} \mathrm{~s}$ for the electronic fluorescence and $10^{-1} \mathrm{~s}$ for the infrared emission). Therefore this mechanism dominates the band width associated with a Lorentzian profile. As the vibrational energy increases, this band width becomes larger (Ionov et al. 1988; Joblin et al. 1995). It is found to increase linearly with temperature, a behaviour which is well-explained by theory.

\subsubsection{Anharmonic shifts and hot bands}

Measurements on gas-phase PAHs have also revealed the dependence on temperature of the IR band positions (Joblin et al. 1995). The positions are shifted towards lower frequencies as the temperature increases, following a linear law. This effect has a similar origin as the homogeneous band width described above. Due to the coupling between modes (intermode anharmonicity), the IR band associated with the mode $i$ is shifted relative to the frequency $\nu_{i}(0)$, that can only be attained when all the other modes are not populated. This temperature shift induces a consequent broadening of the total band emitted during the cooling of PAHs, as was shown in the case of the $3.3 \mu \mathrm{m}$ feature by Joblin et al. (1995).

Another cause of broadening is due to the anharmonicity of the modes themselves (intramode anharmonicity; hot bands as described by Barker et al. 1987). Indeed the transitions $v \rightarrow v-1$ with $v \geq 2$ are usually shifted relative to the fundamental $1 \rightarrow 0$ transition. As a consequence, if the molecule is sufficiently heated to populate the levels $v \geq 2$, the hot bands can create an additional asymmetry in the total emitted feature.

\subsubsection{Rotational broadening}

The values of the rotational constants of PAHs are very small. For instance, the value of $\mathrm{B}$ for coronene is $0.011123 \mathrm{~cm}^{-1}$ (Cossart-Magos \& Leach 1990). It is smaller for larger PAHs since the rotational constant is expected to scale as the inverse of the square of the carbon number (Le Coupanec et al. 1998). As a result, the rotational sub-structure is blurred by the other broadening mechanisms. Only a global rotational envelope can be observed. This is illustrated for instance by the gas-phase spectrum of coronene at $770 \mathrm{~K}$ (Joblin et al. 1995).

The width of the rotational envelope can be quantified by the separation of the maxima of the $P$ and $R$ branches.

$\Delta \nu_{\text {rot }}=4 \sqrt{B k T_{\text {rot }}}$.

Calculations of the rotational temperature of interstellar PAHs led to an average value of $100 \mathrm{~K}$ (Le Coupanec et al. 1998). According to Eq. (6), the total rotational width is therefore $2.4 \mathrm{~cm}^{-1}$ for $\mathrm{C}_{24} \mathrm{H}_{12}$ and becomes inferior to $1 \mathrm{~cm}^{-1}$ for large molecules $\left(N_{\mathrm{C}} \geq 50\right)$. The contribution of the rotational width was therefore neglected in the calculations.

\subsubsection{Molecular diversity}

In this model, we have assumed that all PAHs have exactly the same IR active modes. However, the exact positions of the modes are expected to change from one PAH to the other and therefore to provide an additional broadening to the observed spectra. This is well-known for small molecules $\left(N_{\mathrm{C}} \leq 32\right)$ but has still to be studied for larger systems $N_{\mathrm{C}}$ up to a few hundreds. The possible influence of molecular diversity on the calculated spectrum is discussed in Sect. 3.3.

\subsection{Laboratory and observational inputs}

In the calculations, we aim to use as much as possible the photophysical properties of PAHs derived from laboratory measurements. As mentioned earlier, the relative intensities of the AIBs are more consistent with the carriers being PAH cations rather than neutrals (Szczepanski \& Vala 1993; DeFrees et al. 1993; Schutte et al. 1993; Hudgins \& Allamandola 1995; Langhoff 1996; Cook \& Saykally 1998). Laboratory data on PAH cations were therefore used for the IR band strengths. For the band profiles, only data on neutral PAHs are available. In all cases, the data concern 24-32 carbon PAHs and we had to assume that the derived properties can be extrapolated to larger systems $(\sim 100 \mathrm{C})$.

\subsubsection{Specific heat}

The exact calculation of the specific heat $C(T)$ or the internal energy $U(T)$ requires for each molecule the whole set of vibrational frequencies. To simplify, three typical frequencies have been associated with the $\mathrm{C}-\mathrm{C}$ and $\mathrm{C}-\mathrm{H}$ modes. The frequencies involving $\mathrm{H}$ atoms were readily determined as those of the three IR active $\mathrm{C}-\mathrm{H}$ modes at 3050,1150 and $885 \mathrm{~cm}^{-1}$. For the C-C bonds, three representative frequencies at 1499,840 and $301 \mathrm{~cm}^{-1}$ were derived from the set of modes of dehydrogenated coronene (Boissel et al. 1997). Considering that the total number of modes $3 N-6$ can be split into $3 N_{\mathrm{H}} \mathrm{C}-\mathrm{H}$ modes, and $3\left(N_{\mathrm{C}}-2\right)$ C-C modes, Eq. (3) can then be written as:

$$
\begin{aligned}
U(T)= & N_{\mathrm{H}} \times \sum_{i=1}^{3} \frac{h \nu_{i \mathrm{H}}}{\exp \left(h \nu_{i \mathrm{H}} / k T\right)-1} \\
& +\left(N_{\mathrm{C}}-2\right) \times \sum_{i=1}^{3} \frac{h \nu_{i \mathrm{C}}}{\exp \left(h \nu_{i \mathrm{C}} / k T\right)-1} .
\end{aligned}
$$

\subsubsection{UV absorption}

In the interstellar medium, PAHs are excited by UV photons from stars. In this paper, we have considered the particular case of the planetary nebula IRAS $21282+5050$. According to Cohen \& Jones (1987), the nebula is powered 


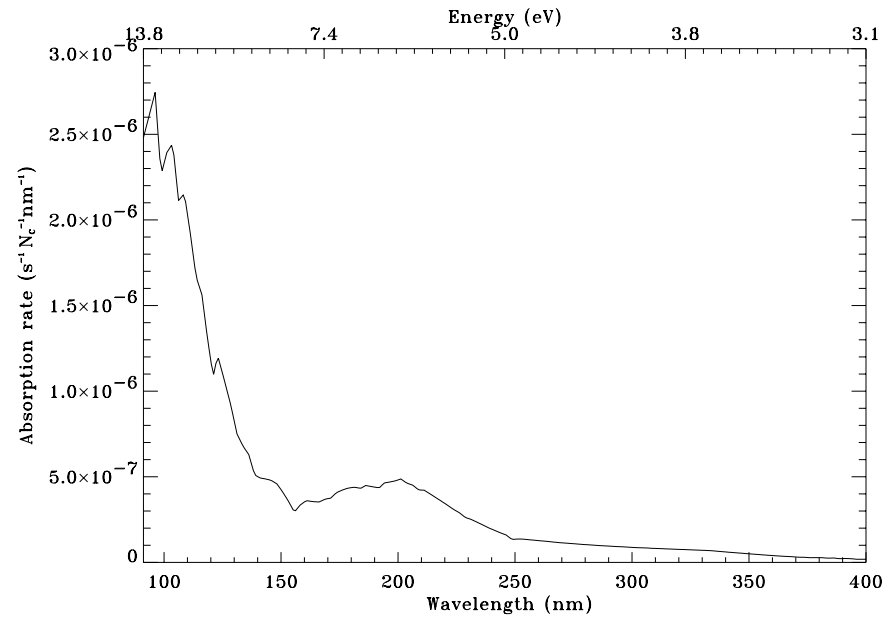

Fig. 1. Absorption rate by PAHs in IRAS 21282+5050 (see Sect. 2.3.2).

by a central star of spectral type O7(f)-[WC11]. The flux $F_{\nu}$ from the star was taken from Kurucz (1979) assuming a stellar temperature of $28000 \mathrm{~K}$ (Leuenhagen \& Hamann 1998). A data compilation on protoplanetary nebulae (Bujarrabal et al. 2001) gives the distance $(D=3 \mathrm{kpc})$ and the total luminosity $\left(L=5.3 \times 10^{3} L_{\odot}\right)$ of this object. The maximum PAH emission is located at about $1^{\prime \prime}$ from the central source (Meixner et al. 1993), e.g. at a projected distance of the central source $d_{*}=0.0145 \mathrm{pc}$. This corresponds to a dilution factor of $W_{\text {dil }}=2.44 \times 10^{-11}$ and an integrated UV photon flux of $G=\int_{6 \mathrm{eV}}^{13.6 \mathrm{eV}} F_{\nu} \mathrm{d} \nu=$ $3.7 \times 10^{5} G_{\mathrm{O}}$, where $G_{\mathrm{O}}$ is the average value for the interstellar medium $\left(G_{\mathrm{O}}=1.6 \times 10^{-10} \mathrm{~W} \mathrm{~cm}^{-2}\right.$; Habing 1968). The rate of photons absorbed by PAHs is given by:

$R_{\mathrm{abs}}\left(N_{\mathrm{C}}^{-1} \mathrm{~s}^{-1}\right)=\int \sigma_{\nu} \frac{F_{\nu}}{h \nu} \mathrm{d} \nu$

where $\sigma_{\nu}$ is the UV absorption cross-section of PAHs.

We have used the UV absorption cross-section $\sigma_{\nu}$ measured by Joblin et al. (1992) on mixtures of neutral PAHs. This cross section consists of a far-UV rise, a broad feature centred at about $210 \mathrm{~nm}$ and a tail extending down to $400 \mathrm{~nm}$. There is not much data on the UV crosssection of PAH cations. One study performed in boron oxide matrices (Robinson et al. 1997) shows that PAH cations are likely to have the same far-UV rise as neutral PAHs. In addition, PAH cations have also relatively sharp absorption bands in the visible part of their spectrum whose intensities and positions depend on the considered species (Salama et al. 1995; Salama et al. 1999). However, in the environment of a hot star, as considered here, the absorption in the UV is largely dominant. The visible bands of PAH cations were therefore not included and the UV-visible cross section of a mixture of neutral PAHs was used (Fig. 1c from Joblin et al. 1992). As shown by the authors, this UV cross section is proportional to the number of carbon atoms. Figure 1 displays the absorption rate of photons by PAHs in the environment of
IRAS $21282+5050$ as a function of wavelengths. The total absorption rate is $R_{\mathrm{abs}}=2.65 \times 10^{-4} N_{\mathrm{C}}^{-1} \mathrm{~s}^{-1}$.

\subsubsection{The vibrational spectrum}

To calculate the IR spectrum emitted by a population of PAHs, it is necessary to know their vibrational spectra including the band strengths (in terms of Einstein coefficients) as well as the band positions and widths and their evolution with temperature.

\section{Band strengths}

The IR vibrational spectra of many $\mathrm{PAHs}^{+}$of sizes up to 32 carbon atoms are known. We have therefore considered the two largest compact cations: coronene $\mathrm{C}_{24} \mathrm{H}_{12}^{+}$and ovalene $\mathrm{C}_{32} \mathrm{H}_{14}^{+}$which have been studied experimentally (Szczepanski \& Vala 1993; Hudgins \& Allamandola 1995) and theoretically (Langhoff 1996). The different IR modes for these species have been gathered together into the main astrophysical features in order to derive Einstein $A_{i}$ coefficients for bands corresponding to the $3.3,6.2$, " 7.7 ", $8.6,11.3$ and $12.7 \mu \mathrm{m}$ AIBs. Classically, the vibrations at 6.2 and "7.7" $\mu \mathrm{m}$ are attributed to $\mathrm{C}-\mathrm{C}$ stretching modes, and the others to $\mathrm{C}-\mathrm{H}$ modes. We note that the spectral range 11.6-13 $\mu \mathrm{m}$ includes the major feature at $12.7 \mu \mathrm{m}$ and a plateau in which minor bands can be observed (Hony et al. 2000). The classical interpretation is that the $11.3 \mu \mathrm{m}$ band is due to solo $\mathrm{H}$ whereas bands at longer wavelengths are attributed to other types of $\mathrm{H}$ (duo, trio, quatro; Allamandola et al. 1989). We did not consider the 11.6-13 $\mu \mathrm{m}$ plateau and put all the intensity into the $12.7 \mu \mathrm{m}$ band. In order to extrapolate the spectra to larger sizes, we assumed that the Einstein coefficients $A_{i}$ are proportional to the number of $\mathrm{C}$ and $\mathrm{H}$ atoms for the $\mathrm{C}-\mathrm{C}$ and $\mathrm{C}-\mathrm{H}$ modes respectively. The spectra of the $\mathrm{PAH}$-like species considered in our model consist therefore of bands at the positions of the interstellar bands with $A_{i}$ coefficients deduced from the $\mathrm{C}_{24} \mathrm{H}_{12}^{+}$and $\mathrm{C}_{32} \mathrm{H}_{14}^{+}$ spectra and scaling with the number of atoms $\left(\mathrm{N}_{\mathrm{C}}\right.$ or $\mathrm{N}_{\mathrm{H}}$; cf. Table 1). These values are quite comparable to those deduced by Schutte et al. (1993) in their standard model. In particular, the cross sections of the $6.2,7.7$ and $8.6 \mu \mathrm{m}$ bands are significantly enhanced compared to those of neutral PAHs.

In addition to the main AIBs, we have also considered two types of bands measured on neutral PAHs but which have not been studied yet on PAH cations. First, the overtone of the $\mathrm{C}-\mathrm{H}$ stretch which has been measured in the laboratory by Joblin (1992) and detected at $1.68 \mu \mathrm{m}$ in the object IRAS $21282+5050$ (Geballe et al. 1994). The Einstein coefficient $A_{20}$ measured for this band is $1 / 6$ of $A_{10}$. Second, PAHs have modes in the far-infrared domain that have to be included in the calculation of the cooling process. Moutou et al. (1996) have reported the far-IR spectra of many neutral PAHs. The positions of 
Table 1. Einstein coefficients for the IR active modes of "interstellar" PAHs. ${ }^{a}$ Generic spectrum based on the spectra of $\mathrm{C}_{24} \mathrm{H}_{12}^{+}$and $\mathrm{C}_{32} \mathrm{H}_{14}^{+}$(Hudgins \& Allamandola 1995; Szczepanski \& Vala 1993 and Langhoff 1996). ${ }^{b}$ Standard model by Schutte et al. (1993).

\begin{tabular}{lcccccc}
\hline \hline Molecule & $\begin{array}{c}A_{3.3 \mu \mathrm{m}} \\
\left(\mathrm{s}^{-1} / N_{\mathrm{H}}\right)\end{array}$ & $\begin{array}{c}A_{6.2 \mu \mathrm{m}} \\
\left(\mathrm{s}^{-1} / N_{\mathrm{C}}\right)\end{array}$ & $\begin{array}{c}A_{\text {“7.7 } \mu \mathrm{m} \mathrm{m}^{\prime \prime}} \\
\left(\mathrm{s}^{-1} / N_{\mathrm{C}}\right)\end{array}$ & $\begin{array}{c}A_{8.6 \mu \mathrm{m}} \\
\left(\mathrm{s}^{-1} / N_{\mathrm{H}}\right)\end{array}$ & $\begin{array}{c}A_{11.3 \mu \mathrm{m}} \\
\left(\mathrm{s}^{-1} / N_{\text {Hsolo }}\right)\end{array}$ & $\begin{array}{c}A_{12.7 \mu \mathrm{m}} \\
\left(\mathrm{s}^{-1} / N_{\text {Hother }}\right)\end{array}$ \\
\hline Generic spectrum $^{a}$ & 6.59 & 2.14 & 5.4 & 0.82 & 5.49 & 0.57 \\
\hline Standard $^{b}$ & 8.95 & 0.93 & 2.68 & 0.82 & 1.87 & 0.58 \\
\hline
\end{tabular}

these bands are quite variable from one molecule to the other. However, for compact PAHs, accumulation points were found at 16, 18.2, 26 and $50 \mu \mathrm{m}$. We have included these bands in the calculations with Einstein coefficients corresponding to the average values measured by Moutou et al. (1996) on compact molecules: $0.057,1.4 \times 10^{-2}$, $1.5 \times 10^{-3}$ and $1.5 \times 10^{-2}\left(\mathrm{~s}^{-1} / N_{\mathrm{C}}\right)$ for the $A_{16 \mu \mathrm{m}}$, $A_{18.2 \mu \mathrm{m}}, A_{26} \mu \mathrm{m}$ and $A_{50 \mu \mathrm{m}}$ coefficients respectively.

\section{Widths and positions of the IR bands}

The temperature dependence of the band positions and widths was studied on a few gas-phase neutral molecules by Joblin et al. (1995). Due to the coupling between modes, the positions of the IR bands shift towards lower frequencies as the temperature increases and the band widths increase. Both effects appear to be linear at least in the high-temperature range. They are expected to be general, independent of the PAH charge state. Temperature laws for the bands' positions and widths were derived mostly from measurements on neutral coronene (Joblin et al. 1995). Compared to the authors' work, only the laws for the band widths were refined by subtracting the contribution of rotation which was not negligible in these experiments at thermal equilibrium $\left(T_{\text {rot }}=T_{\text {vib }}\right)$. Also, the band widths measured in neon matrices at $4 \mathrm{~K}$ (Joblin et al. 1994) were included before fitting the experimental data points, in order to avoid negative widths at low temperatures. In the case of the $3.3 \mu \mathrm{m}$ band, a third-order polynomial had to be included to fit the data at temperatures lower than $850 \mathrm{~K}$. In all other cases, a linear fit was found to be satisfactory with a maximum error of $10 \%$ for the $6.2 \mu \mathrm{m}$ band at $2000 \mathrm{~K}$ (cf. Fig. 2).

The temperature dependence of the band positions and widths is summarized in Table 2 . The values are those of coronene and derivatives except for the position $\nu_{\mathrm{L}}(0)$ of the $11.3 \mu \mathrm{m}$ band. Indeed, the position of the $\mathrm{C}-\mathrm{H}$ outof-plane bending mode is very sensitive to the number of adjacent $\mathrm{H}$. Coronene has only duo $\mathrm{H}$, whereas the $11.3 \mu \mathrm{m}$ band corresponds to solo $\mathrm{H}$. We therefore used the value of $\nu_{\mathrm{L}}(0)$ derived from measurements on ovalene $\mathrm{C}_{32} \mathrm{H}_{14}$ which contains 2 hydrogens solo (Joblin 1992; Joblin et al. 1994). The $6.2 \mu \mathrm{m}$ band width of coronene was measured to be rather constant with temperature, a behaviour which was peculiar in all the measurements reported by Joblin et al. (1995) and could be due to the high symmetry of the molecule. We therefore used for this band the width
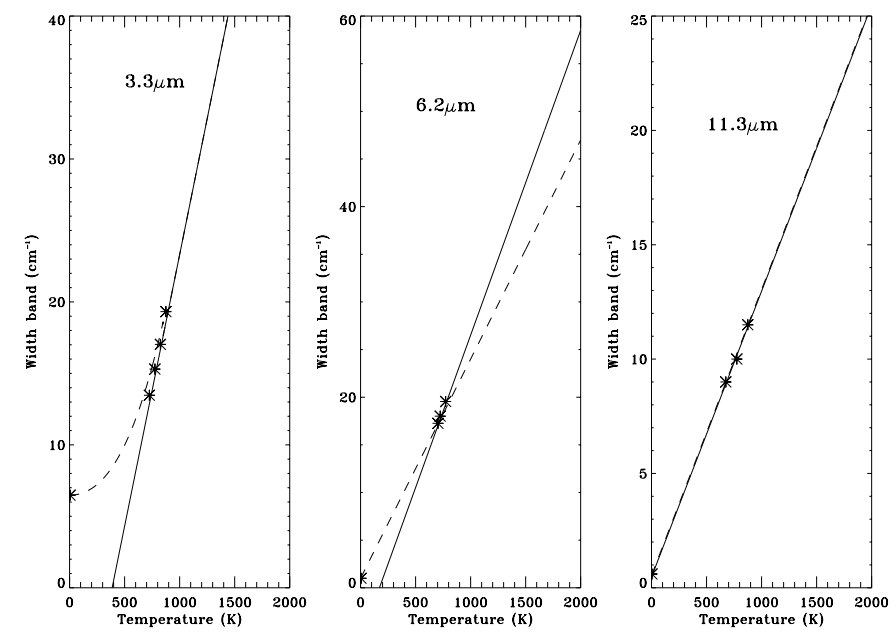

Fig. 2. Widths of the 3.3, 6.2 and $11.3 \mu \mathrm{m}$ bands as a function of temperature. The points at high temperatures are the measurements in gas-phase by Joblin et al. (1995) from which a rotational width was subtracted (except for the $11.3 \mu \mathrm{m}$ band which has a prominent $Q$ branch). The solid line shows the linear fit of the gas-phase data. To describe the width at low temperatures, the point in Ne matrices at $4 \mathrm{~K}$ (Joblin et al. 1995) was included and new fitting curves were derived (dotted lines).

and position measured for methyl-coronene $\mathrm{CH}_{3}-\mathrm{C}_{24} \mathrm{H}_{12}$, a closely related molecule with lower symmetry (Joblin 1992). The implication of the choice of the coefficients $\nu_{\mathrm{L}}(0), \chi^{\prime}, \triangle \nu(0), \chi^{\prime \prime}$ on the calculated IR spectrum is further discussed in Sect. 3.1.

Table 2 also includes the anharmonic shifts used to calculate the positions of the hot bands (transitions $v \rightarrow$ $v-1$, with $v \geq 2$ ) according to:

$\nu_{i, v}(T)=\nu_{i, 1}(T)-(v-1)\left(\nu-\nu_{\mathrm{HB}}\right)$.

For the $3.3 \mu \mathrm{m}$ band, an anharmonicity of $120 \mathrm{~cm}^{-1}$ has been measured by Geballe et al. (1994). No measurements are available for the 6.2 and $11.3 \mu \mathrm{m}$ bands. A value of $5 \mathrm{~cm}^{-1}$ was assumed according to Barker et al. (1987). Concerning the widths, we used the same total width for the hot band of the $3.3 \mu \mathrm{m}$ band as that measured for pyrene $\mathrm{C}_{16} \mathrm{H}_{10}$ at $1.68 \mu \mathrm{m}$ on the $2-0$ overtone transition $\left(\triangle \nu=74 \mathrm{~cm}^{-1}\right.$; Joblin 1992) and also observed in IRAS $21282+5050$ (Geballe et al. 1994). In the case of the $6.2 \mu \mathrm{m}$ and $11.3 \mu \mathrm{m}$ hot bands, there is no experimental data and we assumed that the hot bands follow the same evolution with temperature as the fundamental vibrations. Hot bands have not been considered for 
Table 2. Empirical laws for the IR band positions and widths as a function of temperature: $\nu(T)=\nu_{\mathrm{L}}(0)+\chi^{\prime} T$ and $\Delta \nu(T)=$ $\Delta \nu(0)+\chi^{\prime \prime} T$, derived from measurements on neutral PAHs (a) $\mathrm{C}_{24} \mathrm{H}_{12}$, (b) $\mathrm{C}_{24} \mathrm{H}_{11}-\mathrm{CH}_{3}$ and (c) $\mathrm{C}_{32} \mathrm{H}_{14}$ (Joblin 1992; Joblin et al. 1995 and Fig. 2 in this work). $(*)$ In the case of the $3.3 \mu \mathrm{m}$ band, the $\Delta \nu(0)$ value represents the value at the origin of the linear domain at high temperature $(T>850 \mathrm{~K})$. For $T=[0,850 \mathrm{~K}]$, we used a third-order polynomial $6.48+10^{-5} T^{2}+8 \times$ $10^{-9} T^{3}$. The hot band (transition $v \rightarrow v-1$ with $v \geq 2$ ) positions are derived according to Eq. (9) using the shift $\nu-\nu_{\mathrm{HB}}$; (d) Geballe et al. (1994), (e) Barker et al. (1987).

\begin{tabular}{|c|c|c|c|c|c|}
\hline \multirow[b]{2}{*}{ Band } & \multicolumn{2}{|c|}{$\begin{array}{c}\text { Band position } \\
\left(\mathrm{cm}^{-1}\right)\end{array}$} & \multicolumn{2}{|c|}{$\begin{array}{l}\text { Band width } \\
\left(\mathrm{cm}^{-1}\right)\end{array}$} & \multirow{2}{*}{$\begin{array}{c}\begin{array}{l}\text { Hot band shift } \\
\left(\mathrm{cm}^{-1}\right)\end{array} \\
\nu-\nu_{\mathrm{HB}}\end{array}$} \\
\hline & $\overline{\nu_{\mathrm{L}}(0)}$ & $\overline{\chi^{\prime}}$ & $\Delta \nu(0)$ & $\chi^{\prime \prime}$ & \\
\hline $3.3 \mu \mathrm{m}$ & $3076(\mathrm{a})$ & $-3.2 \times 10^{-2}(\mathrm{a})$ & $-14.69(*)$ & $3.8 \times 10^{-2}(*)$ & $120(\mathrm{~d})$ \\
\hline $6.2 \mu \mathrm{m}$ & 1627 (b) & $-3.8 \times 10^{-2}(\mathrm{~b})$ & $1.00(\mathrm{~b})$ & $2.30 \times 10^{-2}(\mathrm{~b})$ & $5(\mathrm{e})$ \\
\hline $7.7 \mu \mathrm{m}$ & $1326(\mathrm{a})$ & $-2.4 \times 10^{-2}(\mathrm{a})$ & $0.56(\mathrm{a})$ & $8.3 \times 10^{-3}(\mathrm{a})$ & - \\
\hline $8.6 \mu \mathrm{m}$ & $1141(\mathrm{a})$ & $-8.4 \times 10^{-3}(\mathrm{a})$ & $0.66(\mathrm{a})$ & $1.14 \times 10^{-2}(\mathrm{a})$ & - \\
\hline $11.3 \mu \mathrm{m}$ & $896(\mathrm{c})$ & $-2.3 \times 10^{-2}(\mathrm{a})$ & $0.54(\mathrm{a})$ & $1.24 \times 10^{-2}(\mathrm{a})$ & $5(\mathrm{e})$ \\
\hline
\end{tabular}

the 7.7 and $8.6 \mu \mathrm{m}$ bands since several bands fall in the same region (Verstraete et al. 2001) which makes a detailed analysis of individual profiles very difficult.

\subsection{Model of a PAH-like species population}

The IR emission of a PAH-like species population is considered here. This population is defined by the generic formula $\mathrm{C}_{6 \mathrm{p}^{2}} \mathrm{H}_{6 \mathrm{p}}$ and a continuous size distribution given by a power law $N_{\mathrm{C}}^{-\beta}$ between $N_{\mathrm{Cmin}}$ and $N_{\mathrm{Cmax}}$. The formula $\mathrm{C}_{6 \mathrm{p}^{2}} \mathrm{H}_{6 \mathrm{p}}$ corresponds to compact species when $\mathrm{p}$ is an integer (Omont 1986). We used steps of 2 carbons which correspond in the calculations to a non-integer (non-physical) value of $N_{\mathrm{H}}$.

In the following, the notation $\left\{\mathrm{C}_{6 \mathrm{p}^{2}} \mathrm{H}_{6 \mathrm{p}}\right\}$ is given for the PAH-like species of formula $\mathrm{C}_{6 \mathrm{p}^{2}} \mathrm{H}_{6 \mathrm{p}}$ and whose photophysical properties are described above. When considering a size distribution, the three parameters: $N_{\mathrm{Cmin}}, N_{\mathrm{Cmax}}$ and $\beta$ are reported. For instance, values of $N_{\text {Cmin }}=20$, $N_{\text {Cmax }}=178$ and $\beta=2$ were used by Désert et al. (1990) in their model. Finally, we assumed that $1 / 3$ of hydrogen atoms are solo following Schutte et al. (1993).

\subsection{Summary of calculations}

The internal energy of a given PAH is initialized at the value $U_{\text {in }}=h \nu_{\mathrm{UV}}$ corresponding to the absorption of one UV photon. The peak molecular temperature $T_{\mathrm{p}}$ is calculated from Eq. (3). The molecule then cools down in its different $i$ modes from different $v$ levels according to the emission rates given by Eq. (4). At each step $\delta T=1 \mathrm{~K}$, the internal energy changes by $\delta U$ and the fraction of energy emitted in the transition $v \rightarrow v-1$ of the $i$ mode is given by:

$\delta E_{i, v}(T)=\frac{k_{\mathrm{IR}, v}^{i}}{\sum_{i, v} k_{\mathrm{IR}, v}^{i}} \delta U$.

This energy is associated with a band of Lorentzian profile centered at $\nu_{i, v}(\mathrm{~T})$ and of width $\Delta \nu_{i, v}(\mathrm{~T})$. The total energy emitted in the $\mathrm{i}^{\text {th }}$ band $E_{i}\left(U_{\text {in }}\right)$ as well as the total band profile $\psi_{i}\left(U_{\text {in }}\right)$ are obtained by adding all the individual contributions during the cooling of the molecule from $T_{\mathrm{p}}$ to a final temperature $T_{\mathrm{f}}$. In the calculations $T_{\mathrm{f}}$ has been taken at $0 \mathrm{~K}$ but the energy emitted in the midIR for $T$ below $\sim 50 \mathrm{~K}$ is negligible. In a second step, the integration over the absorbed photon distribution is performed (see Sect. 2.3.2 and Fig. 1). The last step consists in integrating over the size distribution of PAHs as described in Sect. 2.4.

\section{Results and discussion}

\subsection{IR emission by individual PAH-like species}

The IR intensities emitted by the PAH-like species $\left\{\mathrm{C}_{6 \mathrm{p}^{2}} H_{6 \mathrm{p}}\right\}$ during their cooling in the environment of the object IRAS $21282+5050$ are presented in Fig. 3 for four different sizes: $\left\{\mathrm{C}_{24} \mathrm{H}_{12}\right\},\left\{\mathrm{C}_{54} \mathrm{H}_{18}\right\},\left\{\mathrm{C}_{96} \mathrm{H}_{24}\right\}$ and $\left\{\mathrm{C}_{150} \mathrm{H}_{30}\right\}$. For an easier comparison of the relative band intensities with the observations, we have chosen to plot each IR band as Lorentzian functions whose positions and widths have been derived from the observed spectrum (Table 5). Figure 3 shows that the emission in the 3.3 and $6.2 \mu \mathrm{m}$ bands decreases with increasing the PAH size. This is particularly steep in the case of the $3.3 \mu \mathrm{m}$ feature which is very weak in the spectrum of a large molecule such as $\left\{\mathrm{C}_{150} \mathrm{H}_{30}\right\}$. We have also calculated the detailed band profiles for the four PAH-like species, taking into account the broadening mechanisms (Sects. 2.2 and 2.3.3). The profiles of the 11.3, 6.2 and $3.3 \mu \mathrm{m}$ bands are presented in Figs. $4-6$. They were obtained by integrating the emission at each temperature step during the cooling as described in Sect. 2.5. The contribution from the hot bands was constructed, using the anharmonic shifts listed in Table 2. Each band can be described by a width and an asymmetry factor. The latter is defined as the ratio of the half width at half maximum $(H W H M)$ measured on the red side to the $H W H M$ measured on the blue side. The values of the asymmetry factor and of the band width $(F H W M)$ calculated for different $\left\{\mathrm{C}_{6 \mathrm{p}^{2}} \mathrm{H}_{6 \mathrm{p}}\right\}$ species and different values of the anharmonicity coefficients $\chi^{\prime}$ and $\chi^{\prime \prime}$ are 


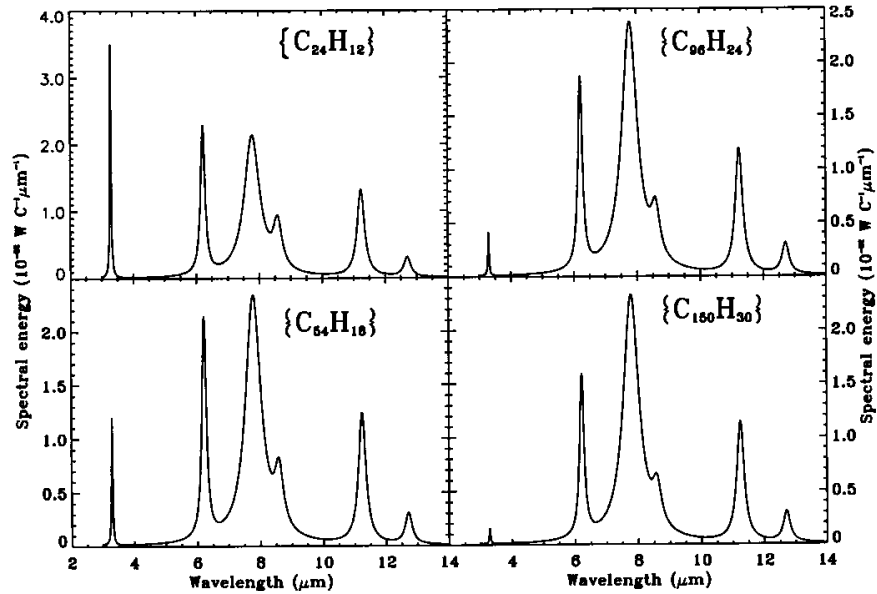

Fig. 3. Calculated IR band intensities emitted by four PAHlike molecules in the environment of IRAS $21282+5050$. The positions and widths of the bands are those of the observed AIBs (cf. Table 5).

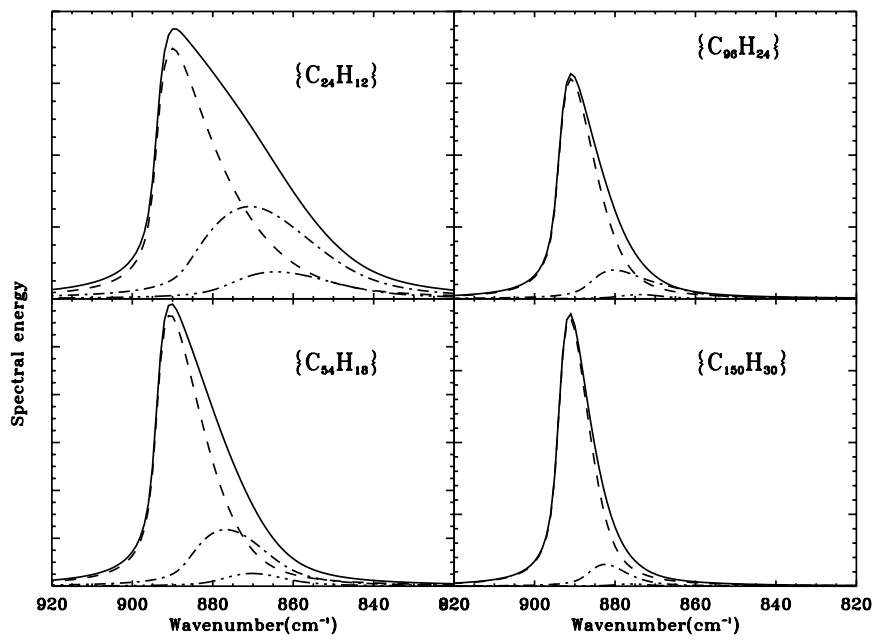

Fig. 4. Calculated $11.3 \mu \mathrm{m}$ band profiles for different PAH-like species (solid line) including the contribution from the fundamental $v=1 \rightarrow 0$ transition (dashed line) and from the hot bands (transitions $2 \rightarrow 1$ and $3 \rightarrow 2$; dot-dashed lines).

displayed in Fig. 7. We observe that the band widths and the asymmetry factors are larger for smaller molecules. The asymmetry is also larger at longer wavelengths (cf. the $11.3 \mu \mathrm{m}$ profile compared to the $3.3 \mu \mathrm{m}$ band profile). The asymmetry of the band profiles is partly due to the hot band contribution. Hot bands are in general limited to the $2 \rightarrow 1$ transition except in the case of the $11.3 \mu \mathrm{m}$ band for which the $3 \rightarrow 2$ transition is still significant for the smallest species (cf. case of $\left\{\mathrm{C}_{24} \mathrm{H}_{12}\right\}$ in Fig. 4). The asymmetry of the $11.3 \mu \mathrm{m}$ band is larger compared to the other bands even without including the contribution from hot bands. This implies that the dominant effect is the shift and broadening of the bands induced by the coupling between modes rather than the anharmonicity of the mode itself. It is therefore interesting to discuss the variations of the band profiles with the values of $\chi^{\prime}$ and $\chi^{\prime \prime}$ which quantify this coupling. The values adopted in the present work

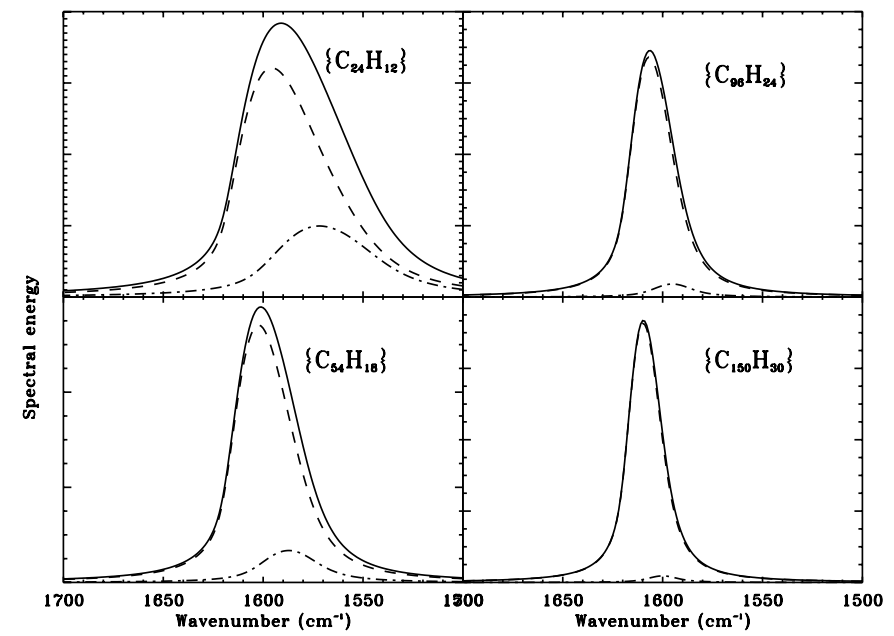

Fig. 5. Calculated $6.2 \mu \mathrm{m}$ band profiles for different PAH-like species (solid line) including the contribution from the fundamental $v=1 \rightarrow 0$ transition (dashed line) and from the hot band (transition $2 \rightarrow 1$; dot-dashed line).

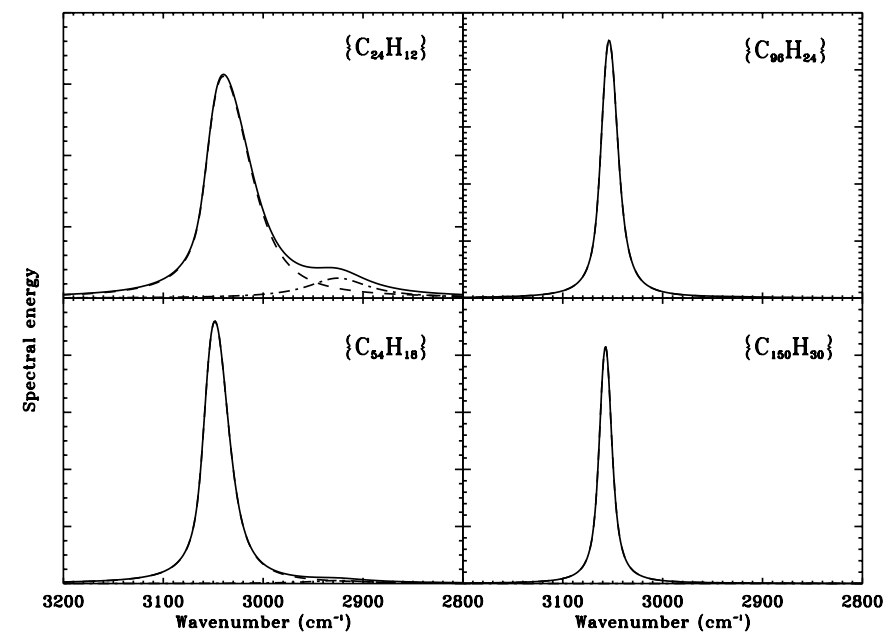

Fig. 6. Calculated $3.3 \mu \mathrm{m}$ band profiles for different PAH-like species (solid line) including the contribution from the fundamental $v=1 \rightarrow 0$ transition (dashed line) and from the hot band (transition $2 \rightarrow 1$; dot-dashed line).

can be questioned on several points. First, the dependence of the band position and width with temperature is linear only at high temperatures. In the low temperature range, the regime differs. Defining a linear law over all the temperature range can therefore induce some errors. For the band width, this happens at higher temperature and we determined the linear law by including the value at $4 \mathrm{~K}$ to avoid negative values (cf. Fig. 2). The resulting error in the high-temperature range is at maximum $10 \%$ for the $6.2 \mu \mathrm{m}$ band at $2000 \mathrm{~K}$ (Sect. 2.3.3) and differences of a few percents are excepted on the calculated profiles. For the band positions, the non-linear behaviour is effective only below $\sim 200 \mathrm{~K}$ (cf. Joblin et al. 1995) and has no or very little impact on the calculated profiles. A second and probably more important effect is that the values of $\chi^{\prime}$ and $\chi^{\prime \prime}$ were derived from the spectrum of a specific molecule: coronene 

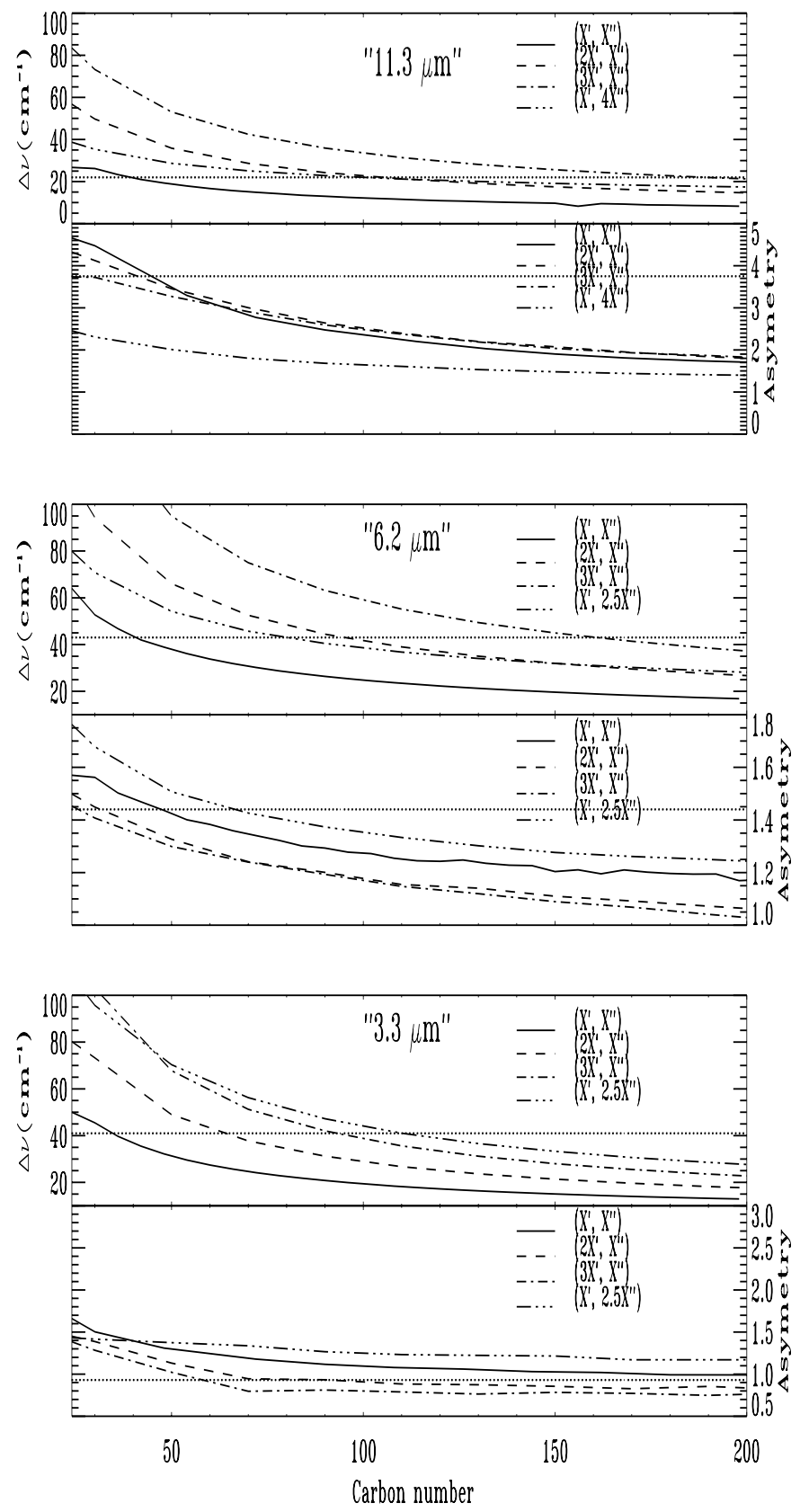

Fig. 7. Dependence of the band width and asymmetry factor on the molecular size and on the values of the anharmonicity coefficients $\chi^{\prime}$ and $\chi^{\prime \prime}$. From top to bottom: the 11.3, 6.2 and $3.3 \mu \mathrm{m}$ bands. The horizontal dotted lines represent the values observed in IRAS $21282+5050$.

and its methylated derivative. Different values for these coefficients would lead to a different band width and asymmetry. To search for a dependence of $\chi^{\prime}$ and $\chi^{\prime \prime}$ with molecular size, we looked more carefully at the available measurements on pyrene $\left(\mathrm{C}_{16} \mathrm{H}_{10}\right)$, coronene $\left(\mathrm{C}_{24} \mathrm{H}_{12}\right)$ and ovalene $\left(\mathrm{C}_{32} \mathrm{H}_{14}\right)$ (Joblin et al. 1995 and Joblin 1992). The comparison was only possible for the $\mathrm{C}-\mathrm{H}$ bands which are the most intense in the IR spectra of PAH neutrals. All the $\chi^{\prime \prime}$ coefficients were derived after extracting the rotational width (cf. 2.3.3). The values of $\chi^{\prime}$ and $\chi^{\prime \prime}$ are plotted in Fig. 8 as a function of size. Maximal variations

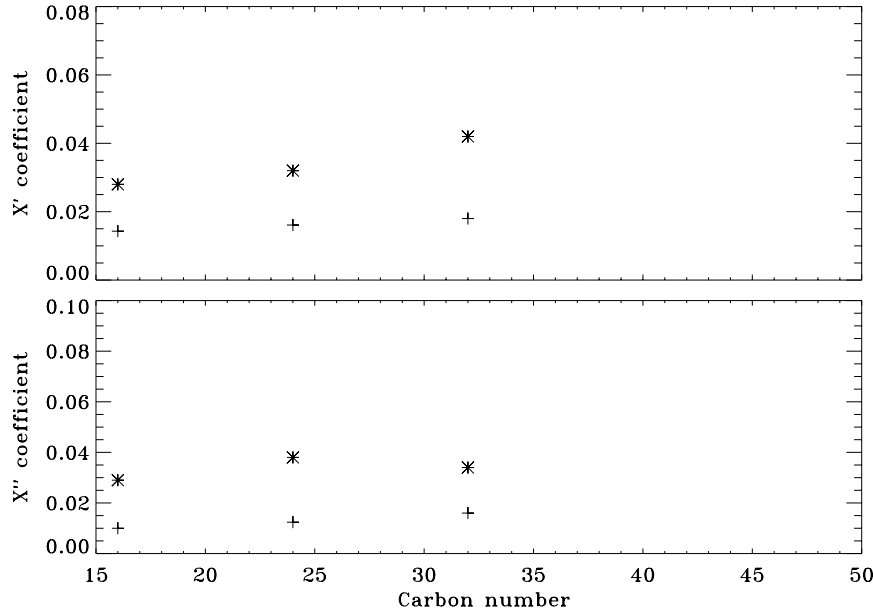

Fig. 8. Evolution of the anharmonicity coefficients $\chi^{\prime}$ and $\chi^{\prime \prime}$ with molecular size for the C-H stretch band (stars) and for the C-H out-of-plane bending band (extracted from the laboratory measurements of Joblin et al. 1995).

of $20 \%$ were found between the three molecules. Although the considered sample is quite restrictive in size, the data suggest an increase of $\chi^{\prime}$ and $\chi^{\prime \prime}$ with the molecular size. Linearly extrapolated to a size of 100 carbon atoms, the value of $\chi^{\prime}$ would be larger by a factor of 2 and 3 for the 3.3 and $11.3 \mu \mathrm{m}$ bands respectively. This is likely to be an upper limit since a saturation in the increase is expected to occur at the solid-state limit. In the case of $\chi^{\prime \prime}$, the factors are 2.5 and 4 respectively. The effect of increasing the values of $\chi^{\prime}$ or $\chi^{\prime \prime}$ on the band width and asymmetry is illustrated in Fig. 7. The band width is found to increase with $\chi^{\prime}$ and $\chi^{\prime \prime}$ with a dominant effect of $\chi^{\prime}$. The asymmetry factor behaves differently. An increase of $\chi^{\prime}$ leads to a significant decrease of the asymmetry in the case of the 3.3 and $6.2 \mu \mathrm{m}$ bands. On the other hand, the asymmetry of the $11.3 \mu \mathrm{m}$ band is slightly modified. This has to do with the difference in the emission regime at short wavelengths compared with that at long wavelengths. Indeed, the variations of the emitted intensity with temperature is slow at $11.3 \mu \mathrm{m}$ but very steep at 3.3 and $6.2 \mu \mathrm{m}$.

\subsection{IR emission by a distribution of PAH-like species}

A size distribution characterized by three parameters: $N_{\text {Cmin }}, N_{\text {Cmax }}$ and $\beta$ was then considered. These parameters can be constrained by using three independent spectral features. As shown by Schutte et al. (1993) and also observed in our calculations, the smallest sizes dominate the emission at short wavelengths. To characterize the minimum size $N_{\text {Cmin }}$, we have therefore considered the width of the $3.3 \mu \mathrm{m}$ band as well as its intensity relative to its overtone $v=2 \rightarrow 0$, detected at $1.68 \mu \mathrm{m}$ in IRAS $21282+5050$ by Geballe et al. (1994). Finally, the $I_{3.3 \mu \mathrm{m}} / I_{11.3 \mu \mathrm{m}}$ ratio was used as a constraint on the average size of the distribution since this ratio strongly depends on the size (cf. Fig. 9). 
Table 3. Calculated values for the $3.3 \mu \mathrm{m}$ band width, $\Delta \nu_{3.3 \mu \mathrm{m}}$, and for the band intensity ratios, $I_{3.3 \mu \mathrm{m}} / I_{11.3 \mu \mathrm{m}}$ and $I_{1.7 \mu \mathrm{m}} / I_{3.3 \mu \mathrm{m}}$, using different size distributions of PAH-like molecules. Comparison with the observations in IRAS $21282+5050$.

\begin{tabular}{cccccc}
\hline \hline Distribution & $\beta$ & $N_{\text {Cmin }}-N_{\text {Cmax }}$ & $\Delta \nu_{3.3} \mu \mathrm{m}\left(\mathrm{cm}^{-1}\right)$ & $I_{3.3 \mu \mathrm{m}} / I_{11.3 \mu \mathrm{m}}$ & $I_{1.68 \mu \mathrm{m}} / I_{3.3 \mu \mathrm{m}}$ \\
\hline $\mathrm{A}$ & 2 & {$[24,200]$} & 37 & 0.18 & $1.1 \times 10^{-2}$ \\
$\mathrm{~B}$ & & {$[24,1000]$} & 37 & 0.14 & $1.1 \times 10^{-2}$ \\
$\mathrm{C}$ & & {$[40,200]$} & 32 & 0.09 & $4.9 \times 10^{-3}$ \\
\hline D & $\mathbf{3 . 5}$ & {$[\mathbf{3 0 , 2 0 0}]$} & $\mathbf{3 8}$ & $\mathbf{0 . 2 0}$ & $\mathbf{9 . 8} \times \mathbf{1 0}^{-3}$ \\
$\mathrm{E}$ & & {$[44,200]$} & 29 & 0.11 & $4.8 \times 10^{-3}$ \\
\hline IRAS 21282+5050 & & 40 & 0.17 & $4.9 \times 10^{-3}$ \\
\hline
\end{tabular}

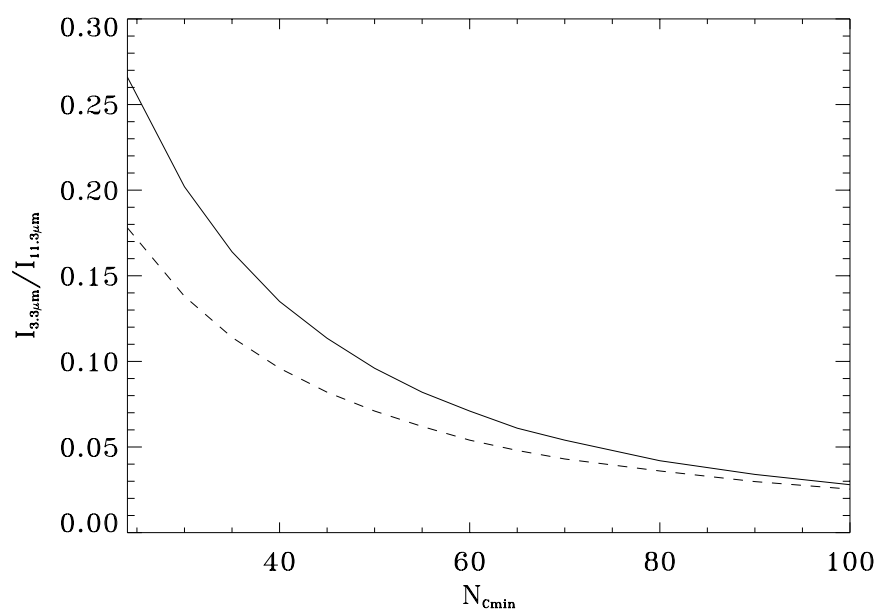

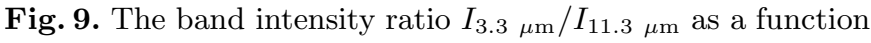
of the minimal size $\left(N_{\mathrm{Cmin}}\right)$ for two size distributions of PAHlike molecules $N_{\mathrm{C}}{ }^{-\beta}$ with $\beta=3.5$ (solid line) and $\beta=2$ (dashed line).

The values of the three selected spectral features: $\Delta \nu_{3.3 \mu \mathrm{m}}, I_{1.68 \mu \mathrm{m}} / I_{3.3 \mu \mathrm{m}}$, and $I_{3.3 \mu \mathrm{m}} / I_{11.3 \mu \mathrm{m}}$ are presented in Table 3 for different size distributions. The first distribution with $\beta=2, N_{\text {Cmin }}=24$ and $N_{\text {Cmax }}=200$ (A in Table 3), which is similar to the one considered by Désert et al. (1990), provides correct values for $\Delta \nu_{3.3 \mu \mathrm{m}}$ and $I_{3.3 \mu \mathrm{m}} / I_{11.3 \mu \mathrm{m}}$. As expected, increasing $N_{\text {Cmax }}$ up to 1000 (B) does not affect the characteristics of the $3.3 \mu \mathrm{m}$ band but simply leads to a decrease of $I_{3.3 \mu \mathrm{m}}$ relative to $I_{11.3 \mu \mathrm{m}}$. In both cases, the calculated $I_{1.68 \mu \mathrm{m}} / I_{3.3 \mu \mathrm{m}}$ ratio is too high by a factor of 2 . The observed value is matched by increasing $N_{\text {Cmin }}$ to 40 (distribution C), but this leads to a $3.3 \mu \mathrm{m}$ band which is too narrow and too weak relative to the $11.3 \mu \mathrm{m}$ one.

Another type of size distribution has been considered with $\beta=3.5$. Such a steep distribution strongly favours the smallest sizes compared to the previous distribution with $\beta=2$ (cf. Fig. 10). A larger value of the minimum size $\left(N_{\mathrm{Cmin}}=30\right.$, distribution $\left.\mathrm{D}\right)$ had therefore to be taken in order to fit $\Delta \nu_{3.3 \mu \mathrm{m}}$ and $I_{3.3 \mu \mathrm{m}} / I_{11.3 \mu \mathrm{m}}$. Here, again, a larger value $N_{\mathrm{Cmin}}=44$ ( $\mathrm{E}$ in Table 3$)$ is required to account for the $1.68 \mu \mathrm{m}$ intensity. It appears then that none of the considered size distributions can fit simultaneously the three selected spectral characteristics. This point is further discussed in the next section.

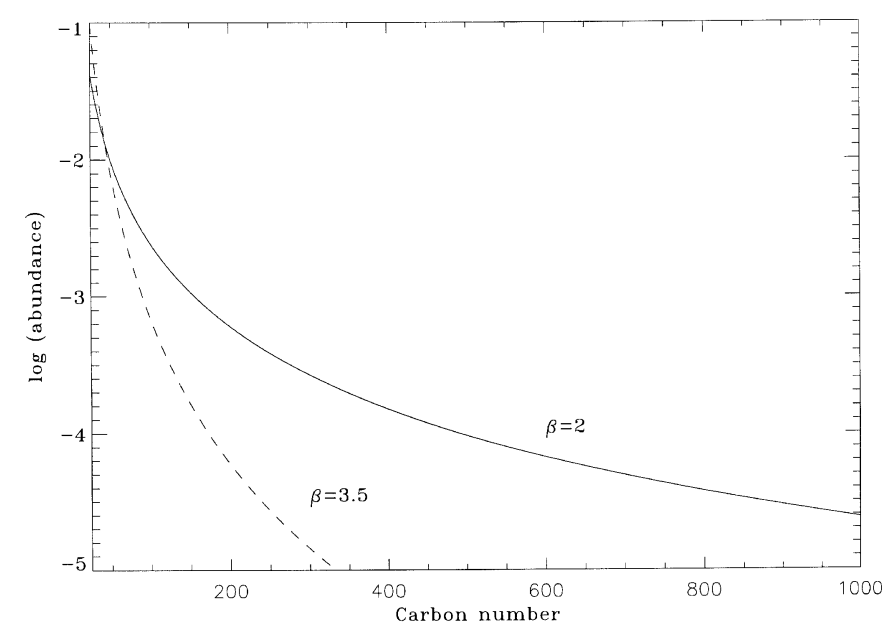

Fig. 10. Normalised abundance for a PAH-like population following a power-law size distribution $N_{\mathrm{C}}^{-\beta}$ with $\beta=2$ and $\beta=3.5$.

Releasing the $I_{1.68 \mu \mathrm{m}} / I_{3.3 \mu \mathrm{m}}$ constraint, we have retained in the following the set of parameters $\mathrm{D}\left(N_{\mathrm{Cmin}}=\right.$ $\left.30, \beta=3.5, N_{\mathrm{Cmax}}=200\right)$, which provides results as good as distribution A without involving too large sizes. Table 4 lists the calculated band intensity ratios relative to the $11.3 \mu \mathrm{m}$ band for the selected size distribution $\mathrm{D}$ in the environment of IRAS $21282+5050$. As can be seen, these ratios match well the observed values. The agreement is especially good for the 6.2 and $3.3 \mu \mathrm{m}$ bands. The largest discrepancy is for the $8.6 \mu \mathrm{m}$ band. This is at least partly due to the difficulty to extract the $8.6 \mu \mathrm{m}$ band from the wing of the strong "7.7" $\mu \mathrm{m}$ feature.

The calculated band profiles for the distribution D are displayed in Figs. 11-13 for the 11.3, 6.2 and $3.3 \mu \mathrm{m}$ bands respectively. The agreement between the calculated and observed bands appears to be nice as can be seen from the figures and from the values of the widths and asymmetry factors (Table 5). In the case of the $11.3 \mu \mathrm{m}$ band, the calculated band falls exactly at the position of the observed band. The observed profile with a steep rise on the blue side and extended wing on the red side, is also very well reproduced by the calculations. This is also the case for the profile of the $6.2 \mu \mathrm{m}$ feature, although the positions of the calculated and observed bands differ by $7 \mathrm{~cm}^{-1}$. Finally, the calculated profile at $3.3 \mu \mathrm{m}$ appears 
Table 4. The relative IR band intensity ratios calculated for the PAH-like population of size distribution D (Table 3) compared with the values measured in IRAS $21282+5050$.

\begin{tabular}{lcccc}
\hline \hline & $I_{3.3 \mu \mathrm{m}} / I_{11.3 \mu \mathrm{m}}$ & $I_{6.2 \mu \mathrm{m}} / I_{11.3 \mu \mathrm{m}}$ & $I_{7.7 \mu \mathrm{m}} / I_{11.3 \mu \mathrm{m}}$ & $I_{8.6 \mu \mathrm{m}} / I_{11.3 \mu \mathrm{m}}$ \\
\hline Calculated spectrum & 0.20 & 1.01 & 3.82 & 0.54 \\
\hline Observed spectrum & 0.16 & 0.98 & 2.62 & 0.79 \\
\hline
\end{tabular}

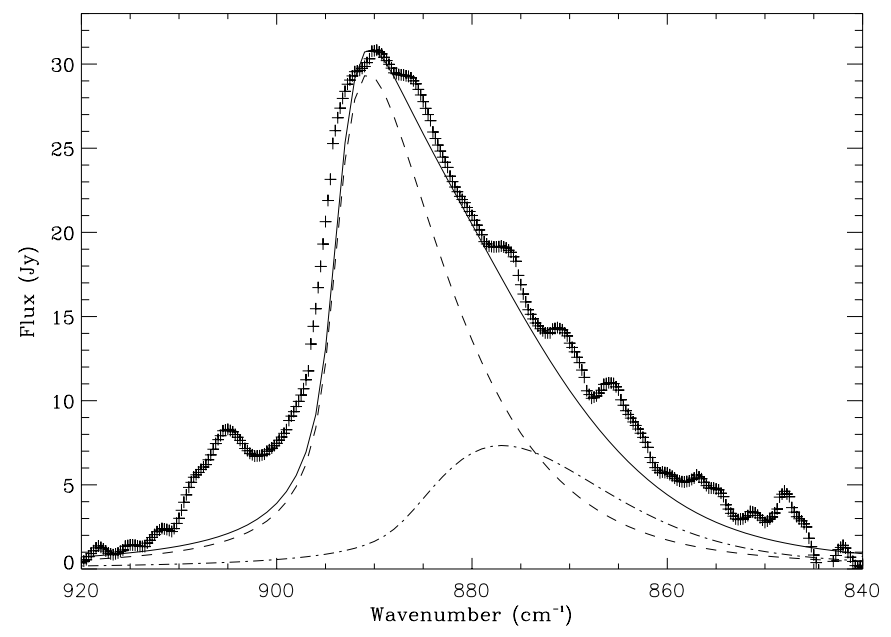

Fig. 11. Calculated $11.3 \mu \mathrm{m}$ band profile (solid line) for the PAH-like population of size distribution D (Table 3) compared with the observations in IRAS $21282+5050$ (crosses). The calculated band includes the fundamental $v=1 \rightarrow 0$ transition (dashed line) and the hot band (transition $2 \rightarrow 1$; dotdashed line).

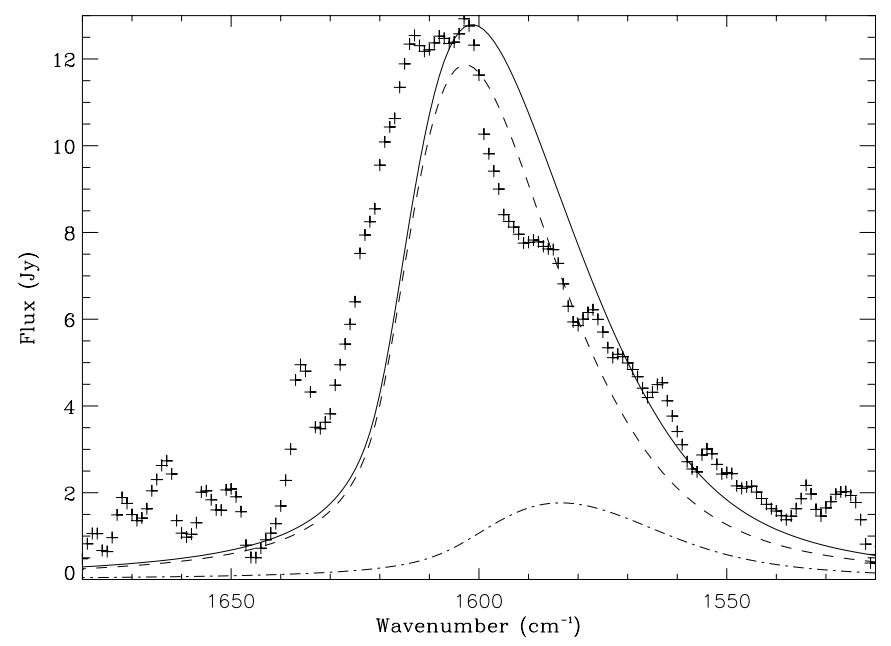

Fig. 12. Calculated $6.2 \mu \mathrm{m}$ band profile (solid line) for the PAH-like population of size distribution D (Table 3) compared with the observations in IRAS 21282+5050 (crosses). The calculated band includes the fundamental $v=1 \rightarrow 0$ transition (dashed line) and the hot band (transition $2 \rightarrow 1$; dotdashed line).

to be the less satisfactory with an asymmetry factor larger than the observed one (Fig. 13).

As previously discussed, the asymmetry of the profiles in the case of individual molecules results from the anharmonicity effects. In the case of a distribution of

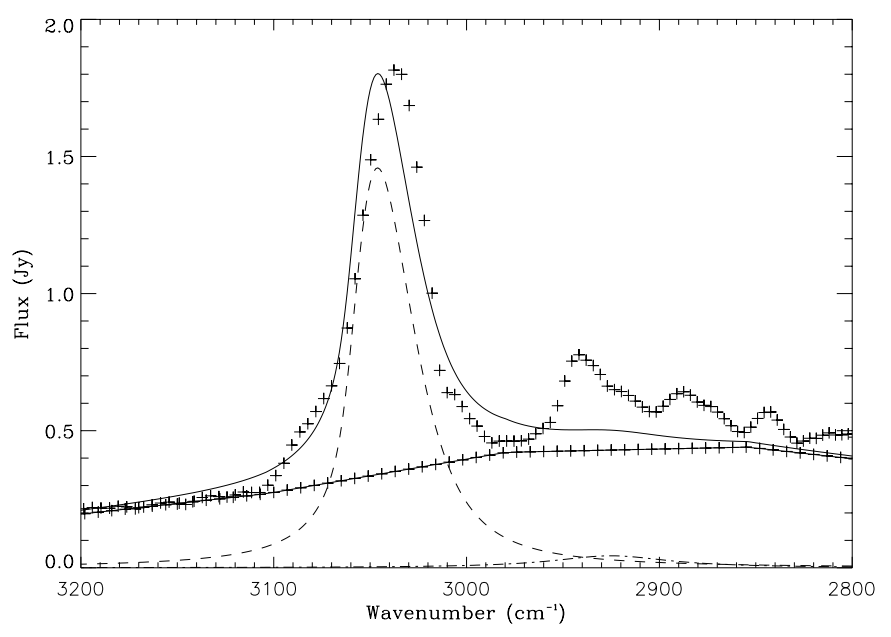

Fig. 13. Calculated $3.3 \mu \mathrm{m}$ band profile (solid line) for the PAH-like population of size distribution D (Table 3) compared with the observations in IRAS $21282+5050$ (crosses). The calculated emission bands in the fundamental $v=1 \rightarrow 0$ transition (dashed line) and in the $v=2 \rightarrow 1$ hot band (dot-dashed line) have been superposed on an underlying continuum represented by linked crosses.

PAH-like species, it also reflects the variety of sizes and therefore of emission temperatures (Verstraete et al. 2001). The profiles obtained for the distribution D can be compared to the profiles of the average size of the distribution, $\left\langle N_{\mathrm{C}}>=48\right.$. As can be seen in Fig. 14, the red wing is more extended when a distribution of molecules is considered. The effect increases for the bands at shorter wavelengths. At $6.2 \mu \mathrm{m}$, an increase of the width and asymmetry of $3 \mathrm{~cm}^{-1}$ and $12 \%$ respectively is observed. The increase is up to $10 \mathrm{~cm}^{-1}$ and $20 \%$ in the case of the $3.3 \mu \mathrm{m}$ band. The increase of asymmetry provided by the distribution leads to a better fit of the AIBs except in the case of the $3.3 \mu \mathrm{m}$ band. This is due to the contribution of the smallest sizes $\left(N_{\mathrm{C}}<48\right)$ that is up to $83 \%$ for the $3.3 \mu \mathrm{m}$ band and $60 \%$ for the $11.3 \mu \mathrm{m}$ band (cf. Fig. 15). Finally, calculations have also been performed for the "7.7" and $8.6 \mu \mathrm{m}$ bands. The "7.7" $\mu \mathrm{m}$ AIB is known to consist of several components (Joblin et al. 2000 and Verstraete et al. 2001). Widths between 20 and $30 \mathrm{~cm}^{-1}$ were extracted for the individual bands. Our calculations provide a value of $21 \mathrm{~cm}^{-1}$ which is consistent with these studies. The $8.6 \mu \mathrm{m}$ band appears to be singular since the calculated band does not account for the observed intensity nor for the observed profile.

The results of our calculations are summarized in Fig. 16. The calculated profiles are displayed for the 3.3, 
Table 5. Peak positions and full widths at half maximum of the emission bands calculated for the PAH-like population of size distribution D (Table 3) compared with the values observed in IRAS 21282+5050.

\begin{tabular}{|c|c|c|c|c|c|c|}
\hline \multirow[b]{2}{*}{ Band } & \multicolumn{3}{|c|}{ Calculations } & \multicolumn{3}{|c|}{ Observations } \\
\hline & $\begin{array}{l}\text { Band position } \\
\left(\mathrm{cm}^{-1}\right)\end{array}$ & $\begin{array}{l}\text { Band } \\
\text { asymmetry }\end{array}$ & $\begin{array}{l}\text { Band width } \\
\left(\mathrm{cm}^{-1}\right)\end{array}$ & $\begin{array}{l}\text { Band position } \\
\left(\mathrm{cm}^{-1}\right)\end{array}$ & $\begin{array}{l}\text { Band } \\
\text { asymmetry }\end{array}$ & $\begin{array}{l}\text { Band width } \\
\left(\mathrm{cm}^{-1}\right)\end{array}$ \\
\hline $3.3 \mu \mathrm{m}$ & 3045 & 1.42 & 38 & 3040 & 0.93 & 41 \\
\hline $6.2 \mu \mathrm{m}$ & 1601 & 1.46 & 42 & 1608 & 1.44 & 43 \\
\hline $7.7 \mu \mathrm{m}$ & 1323 & 1.01 & 21 & " 1283" & - & $" 98 "$ \\
\hline $8.6 \mu \mathrm{m}$ & 1135 & 0.88 & 10 & "1164" & - & $" 45 "$ \\
\hline $11.3 \mu \mathrm{m}$ & 889 & 3.3 & 19 & 889 & 3.75 & 22 \\
\hline
\end{tabular}

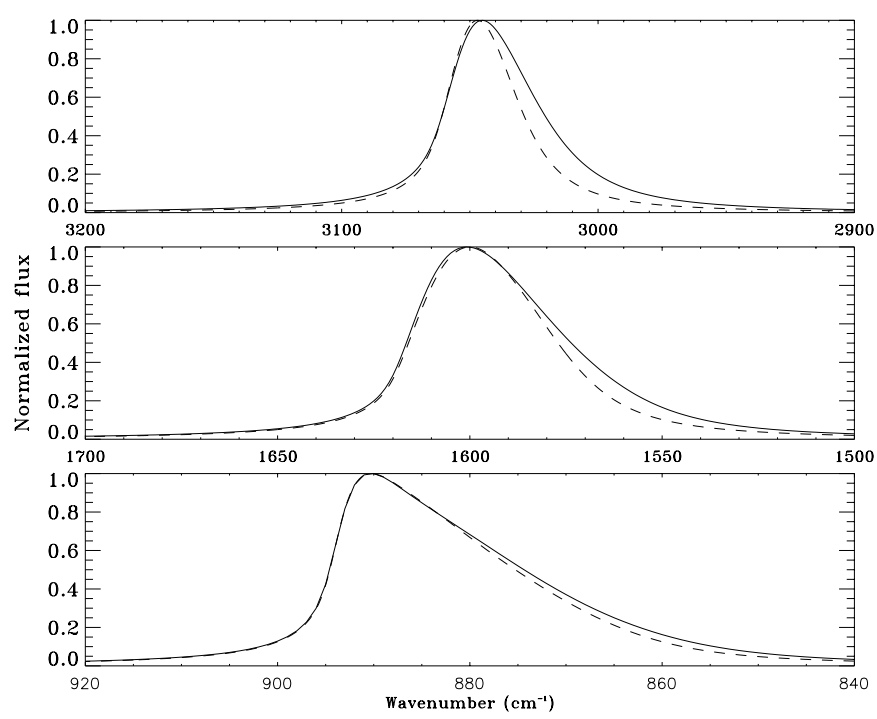

Fig. 14. Comparison of the profiles calculated for the distribution D (solid line) and for a single molecule representing the average size of the distribution $\left\langle N_{\mathrm{C}}\right\rangle=48$. From top to bottom: the $3.3,6.2,11.3 \mu \mathrm{m}$ bands.
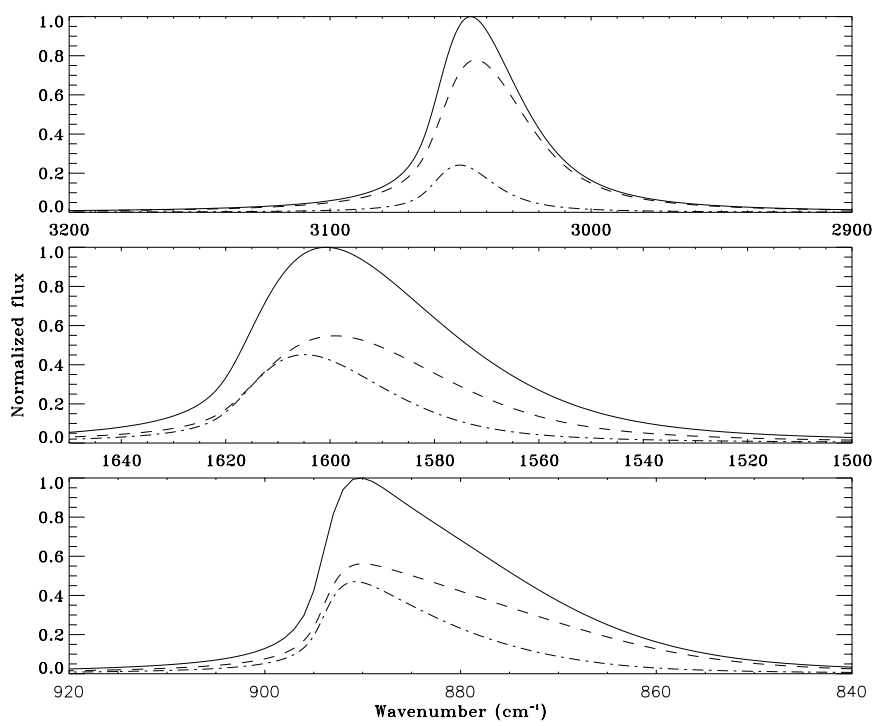

Fig. 15. Contribution of the smallest sizes $\left(30 \leq N_{\mathrm{C}} \leq 48\right.$; dotted lines $)$ and of the largest sizes $\left(50 \leq N_{\mathrm{C}} \leq 200\right.$; dashdotted lines) to the total profiles calculated for distribution $\mathrm{D}$ (solid line). From top to bottom: the 3.3, 6.2, $11.3 \mu \mathrm{m}$ bands.

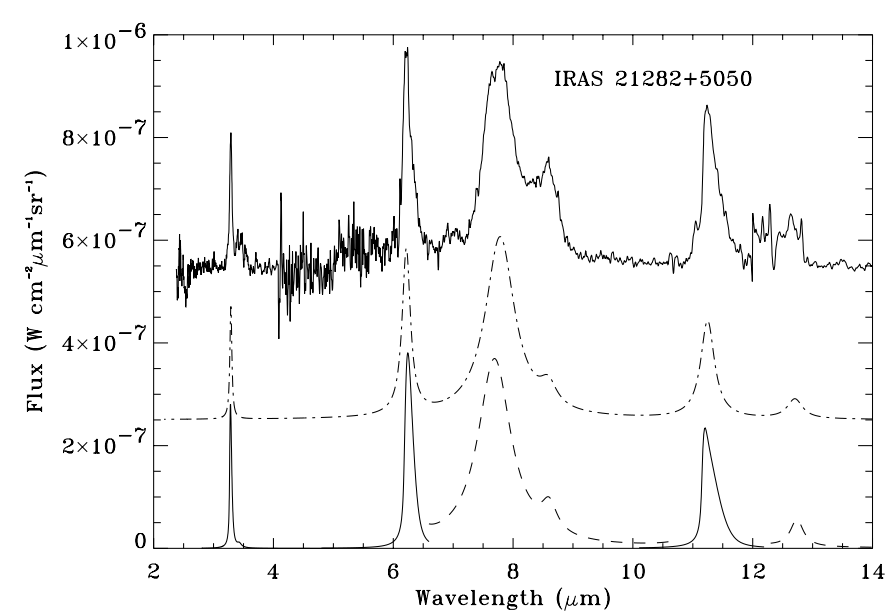

Fig. 16. Summary of calculated spectra from this work compared to the AIB spectrum in IRAS $21282+5050$ (top spectrum; ISO-SWS observations by M. Jourdain de Muizon, L. B. d'Hendecourt, A. Heras and collaborators). The spectra are shifted for clarity with the bottom spectrum corresponding to the distribution D of PAH-like species and the medium spectrum to the average size of the distribution $\left(\left\langle N_{\mathrm{C}}\right\rangle=\right.$ $48 \mathrm{C}$ ). The solid lines correspond to calculated profiles and the others are lorentzian profiles at the observed positions and widths (Table 5).

6.2 and $11.3 \mu \mathrm{m}$ bands. For the other bands (dashed lines), the observed positions and widths (Table 5) were used in order to favour the comparison between the observed and calculated spectra. A remarkable agreement is also found between the calculated and observed IR flux. Absolute values were determined for the calculated spectrum by assuming a total column density of $1.8 \times 10^{21} \mathrm{~cm}^{-2}\left(A_{V}=\right.$ 1) and $10 \%$ of interstellar carbon in PAH species (using $[\mathrm{C} / \mathrm{H}]_{\mathrm{ISM}}=2.610^{-4}$, Snow \& Witt 1996). Finally, we have assumed that the PAH emission is spread over a 3.6" aperture according to the high spatial resolution images of IRAS $21282+5050$ obtained by Meixner et al. (1993).

\subsection{Discussion}

Generic photophysical properties for the population of interstellar PAHs were defined using laboratory data or quantum chemical calculations on small molecules and 
extrapolated to larger sizes. The Einstein coefficients $A_{i}$ are from ionized species whereas the anharmonicity coefficients $\chi^{\prime}$ and $\chi^{\prime \prime}$ are known only for neutrals. Another major assumption we made is that the IR frequencies and their temperature dependence are the same for all the PAHs species. From these hypotheses, the parameters of a distribution $\left(N_{\mathrm{Cmin}}=30 ; \beta=3.5 ; N_{\mathrm{Cmax}}=200\right)$ have been adjusted to match the ratio $I_{3.3 \mu \mathrm{m}} / I_{11.3 \mu \mathrm{m}}$ and the width of the $3.3 \mu \mathrm{m}$ AIB that are observed in the object IRAS $21282+5050$. The calculated spectrum appears to provide a good match of the relative intensities of the AIBs as well as to account for the profiles of the 6.2 and $11.3 \mu \mathrm{m}$ AIBs. The shape of these bands appears to be characteristic of the anharmonicity of molecular modes. Restricted inhomonegeous broadening is caused by the distribution of molecular sizes and therefore of temperatures. No spectral diversity (i.e. change of the IR spectrum from one species to the others) was included. The very good fit obtained for the 6.2 and $11.3 \mu \mathrm{m}$ profiles leaves indeed very little room for such a spectral diversity. Including a dispersion of the central frequencies with the size and the specific geometry of the molecules would lead to a change of the band shape with difficulties to fit the observed band. In our model, the observed band profile is naturally explained. The case of the $3.3 \mu \mathrm{m}$ band appears to be different with an observed profile more symmetric than the calculated one. Several explanations might be tentatively given to account for this discrepancy. Inhomonegeous effects might be more important for this band. As seen previously, the $3.3 \mu \mathrm{m}$ band is dominated by the smallest sizes which are likely to have enhanced spectral diversity compared to larger sizes. Another reason, which might be invoked is the fact that we used values of the anharmonicity coefficients $\chi^{\prime}$ and $\chi^{\prime \prime}$ from neutrals. From the point of view of the Einstein coefficients the $3.3 \mu \mathrm{m}$ band is strongly perturbed by ionisation as shown by quantum chemical calculations (Langhoff 1996 for instance). The dependence of the band shape with temperature might then also be perturbed. As opposed to the other AIBs, the "7.7" $\mu \mathrm{m}$ band is very broad and shows some sub-structures. A decomposition into Lorentzian profiles leads to at least 4 components in this spectral region (Joblin et al. 2000; Verstraete et al. 2001). This might give evidence for spectral diversity. Still, this work underlines the fact that the AIB spectrum does not contain much spectral diversity. In particular, our model was able to match the profile of the 6.2 and $11.3 \mu \mathrm{m}$ bands by assuming that all PAHs in the distribution emit at the same frequencies $\nu_{i}(T)$ when heated at the same temperature $T$. This could mean that the IR spectrum of the emitting species reaches a solid-like limit although these species behave as molecules from a photophysical point of view. An interesting question concerns the size at which such a limit is attained. Another possibility is that interstellar PAHs have similar spectra because they have similar structures. In this model, we have used the formula $\mathrm{C}_{6 \mathrm{p}^{2}} \mathrm{H}_{6 \mathrm{p}}$ which is characteristic of compact PAHs. May be photodissociation gives rise to natural selection among interstellar PAHs, only leaving species with special charac- teristics, for instance compact species. As an illustration Joblin et al. (1997) have shown that the condensed form of the tricoronene $\left(\mathrm{C}_{72} \mathrm{H}_{24}\right.$ compared with the more linear form $\mathrm{C}_{72} \mathrm{H}_{28}$ ) was more stable upon UV laser radiation.

\section{Conclusion}

In this paper, we present a model that can be used to calculate the IR emission spectrum of a population of PAH-like species. Taking account of the photophysical properties of small neutral and ionized species, this model enables to consider, for the first time, not only the intensities of the bands but also their profiles. Such an approach puts stronger constraints on the population of emitting species.

The results that are presented in this paper are very encouraging. They show that a molecular model can account for the relative band intensities as well as for the profiles of the 6.2 and $11.3 \mu \mathrm{m}$ bands. Several points remain however to be elucidated. First, the profile of the $3.3 \mu \mathrm{m}$ band which appears to be more symmetric in the observations than in the calculations. Second and probably more important the apparent lack of spectral diversity in the 6.2 and $11.3 \mu \mathrm{m}$ bands. This certainly gives new information on the interstellar population. However, more studies have to be performed before definitive conclusions can be made. We have discussed the particular case of the planetary nebula IRAS $21282+5050$ but the model has to be applied to many other objects. A recent study by Verstraete et al. (2001) concerned the reflection nebula NGC 2023 and the photodissociation region M17 SW. Also, this work emphasises the need to study the photophysical properties of PAH-like systems containing typically 100 carbon atoms. This is one of the goal of PIRENEA (Joblin et al. 2002), a new experimental setup which is now being developed at the Centre d'Étude Spatiale des Rayonnements in Toulouse.

Acknowledgements. We thank the referee Xander Tielens for helping us to increase the impact that the paper may have for the interpretation of the AIB spectrum.

\section{References}

Allamandola, L. J., Tielens, A. G. G. M., \& Barker, J. R. 1989, ApJS, 71, 733

Andriesse, C. D. 1978, A\&A, 66, 169

Barker, J. R., Allamandola, L. J., \& Tielens, A. G. G. M. 1987, A\&A, 315, L61

Boissel, P., de Parseval, P., Marty, P., et al. 1997, J. Chem. Phys., 106, 1

Borghesi, A., Bussoletti, E., \& Colangeli, L. 1987, ApJ, 314, 422B

Boulanger, F. 1999, in Solid Interstellar Matter: The ISO Revolution, ed. L. d'Hendecourt, C. Joblin, A. Jones (EDP Sciences, Les Ulis), Les Houches Ser., 11, 20

Bujarrabal, V., Castro-Carrizo, A., Alcolea, J., \& Sànchez Contreras, C. 2001, A\&A, 377, 868

Cohen, M., \& Jones, B. F. 1987, ApJ, 321, L151 
Cook, D. J., Schlemmer, S., Balucani, N., et al. 1998, J. Phys. Chem. A, 102, 1465

Cook, D. J., \& Saykally, R. J. 1998, A\&A, 493, 793

Cossart-Magos, C., \& Leach, S. 1990, A\&A, 233, 559

Désert, F. X., Boulanger, F., \& Puget, J. L. 1990, A\&A, 237, 215

DeFrees, D. J., Miller, M. D., Talbi, D., et al. 1993, ApJ, 408, 530

Geballe, T. R., Joblin, C., d'Hendecourt, L. B., et al. 1994, ApJ, 434, L15

Felker, P. M., \& Zewail, A. H. 1984, Chem. Phys. Lett., 108, 303

Habing, H. J. 1968, Bull. Astron. Inst. Netherlands, 19, 421

Hony, S., Van Kerckhoven, C., Peeters, E., et al. 2000, in ISO beyond the Peaks, ESA Pub. Ser., SP-456, 63

Hudgins, D. M., \& Allamandola, L. J. 1995, J. Phys. Chem., 99, 3033

Ionov, S. I., Stuchebryukhov, A. A., Bagratashvili, V. N., et al. 1988, Appl. Phys. B, 47, 229

Joblin, C., Pech, C., Armengaud, M., Frabel, P., \& Boissel, P. 2002, in Infrared and Submillimeter Space Astronomy (EDP Sciences, Les Ulis)

Joblin, C., Abergel, A., Bregman, J., et al. 2000, in ISO beyond the Peaks, ESA Pub. Ser., SP-456, 49

Joblin, C., Masselon, C., Boissel, P., et al. 1997, Rapid. Commun. Mass Spectrom., 11, 1619

Joblin, C., Boissel, P., Léger, A., et al. 1995, A\&A, 299, 835

Joblin, C., d'Hendecourt, L. B., Léger, A., et al. 1994, A\&A, 281,923

Joblin, C., Léger, A., \& Martin, P. 1992, ApJ, 393, L79

Joblin, C. 1992, Ph.D. Thesis, Université Paris 7
Kurucz, R. L. 1979, ApJS, 40, 1

Langhoff, S. R. 1996, J. Phys. Chem., 100, 2819

Le Coupanec, P., Rouan, D., \& Léger, A. 1998, A\&A, 338, 217

Léger, A., d'Hendecourt, L. B., \& Défourneau, D. 1989, A\&A, 216,148

Léger, A., Boissel, P., \& d'Hendecourt, L. B. 1988, Phys. Rev. Lett., 60(10), 921

Léger, A., \& Puget, J. L. 1984, A\&A, 137, L5

Leuenhnagen, U., \& Hamann, W.-R. 1998, A\&A, 330, 265

Meixner, M., Skinner, C. J., Temi, P., et al. 1993, ApJ, 411, 266

Moutou, C., Léger, A., \& d'Hendecourt, L. B. 1996, A\&A, 310, 297

Omont, A. 1986, A\&A, 164, 159

Papoular, R., Conard, J., Giuliano, M., et al. 1989, A\&A, 217, 204

Robinson, M. S., Beegle, L. W., \& Wdowiak, T. J. 1997, ApJ, 474,474

Sakata, A., Wada, S., Onaka, T., et al. 1987, ApJ, 320, L63

Salama, F., Galazutdinov, G. A., Krelowski, J., et al. 1999, ApJ, 526, 265

Salama, F., Joblin, C., \& Allamandola, L. J. 1995, P\&SS, 43, 1165

Schutte, W. A., Tielens, A. G. G. M., \& Allamandola, L. J. 1993, ApJ, 415, 397

Sellgren, K. 1984, ApJ, 277, 623

Snow, T. P., \& Witt, A. N. 1996, ApJ, 468, L65

Szczepanski, J., \& Vala, M. 1993, ApJ, 414, 646

Verstraete, L., Pech, C., Moutou, C., et al. 2001, A\&A, 372, 981 